\title{
Coherent phonon modes in nanostructured zinc oxide synthesized by arc- exploding technique
}

Rohit Medwal $^{1}$, Surbhi Gupta ${ }^{1 *}$, Shojan P. Pavunny ${ }^{1}$, Rajesh K. Katiyar ${ }^{1}$, S. Annapoorni ${ }^{2}$, and R S. Katiyar ${ }^{1 * *}$

1.Department of Physics and Institute for Functional Nanomaterials, University of Puerto Rico, P.O. Box 70377, San Juan, PR 00936-8377, USA

2. Department of Physics and Astrophysics, University of Delhi, Delhi, 110007, India Authors of correspondence*surbhi.gupta208@gmail.com,** rkatiyar@hpcf.upr.edu

\begin{abstract}
:
We report the observation of coherent phonon modes in zinc oxide nanostructures synthesized by arc exploding technique (AET). The band gap $\left(\mathrm{E}_{\mathrm{g}}\right)$ is estimated to be $3.3 \mathrm{eV}$ from UV-absorption spectra. The UV resonance Raman spectra (UVRRS)
\end{abstract}

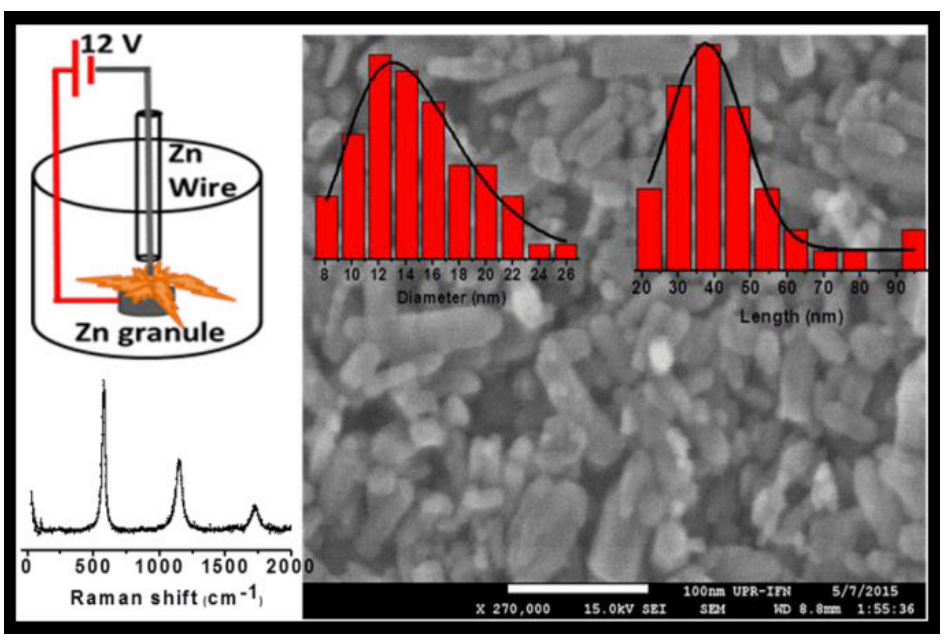
revealed the presence of multiple longitudinal optical (LO) phonon modes demonstrating the transformation of zinc to zinc oxide as a function of ultra-sonication time. The observed multiple $\mathrm{A}_{1}(\mathrm{LO})$ Raman active phonons in $\mathrm{ZnO}$ nanostructures were attributed to atomic displacement parallel to wurtzite c-axis. Higher order harmonics and intensity variations in multiple LO phonon modes were interpreted using cascade model in resonant spectra. The appearance of $\mathrm{E}_{2}$ (low) at $\sim 101 \mathrm{~cm}^{-1}$ was observed with higher processing time along with enhanced multiple LO phonon modes, indicating the oscillations of zinc sub-lattice. The $\mathrm{Zn}$ to $\mathrm{ZnO}$ evolution was also confirmed using Photoluminescence (PL) spectroscopy showing band-edge emission at $380 \mathrm{~nm}$. Keywords: Raman, Zinc Oxide, Nano-crystalline Materials 


\section{Introduction}

Zinc oxide $(\mathrm{ZnO})$ nanoparticles having a wide direct band gap of $\sim 3.2 \mathrm{eV}$ and a large excitonic binding energy of $60 \mathrm{meV}$ is a valuable candidate for scientific community due to its various potential applications in photovoltaic, optoelectronic, biomedical, etc. devices [1-3]. AET is an elegant and simple approach to synthesize nanoparticles of oxide materials even at room temperature [4-5]. AET is a physical, top-down process in which high density plasma is formed between two metal electrodes (anode and cathode). The control on the plasma can be achieved by varying current density in the electrodes by (a) changing the electrode dimension and (b) varying the magnitude of the current flowing through the electrodes. The advantages of opting for AET in this study over other methods are its (a) fast and cost effective processing (b) negligible instrumentation cost (c) better control on the shape and size (d) potential to maintain stoichiometry of the parent materials (e) capability to have chemical free surface as no chemicals was used in the synthesis.

Wurtzite symmetry can be better understood by identifying the available phonon modes in $\mathrm{ZnO}$ wurtzite structure. Based on the group theory, the different phonon modes available in the wurtzite structure are given by $\Gamma=A_{1}+2 B_{1}+E_{1}+2 E_{2}$ and their Raman tensors which provides a detail understanding of phonon dynamics of the $\mathrm{ZnO}$ structure are given as follows [6],

$\mathrm{P}_{\mathrm{A} 1(\mathrm{z})}=\left(\begin{array}{ccc}a & 0 & 0 \\ 0 & a & 0 \\ 0 & 0 & b\end{array}\right), \quad \mathrm{P}_{\mathrm{E} 1(\mathrm{x})}=\left(\begin{array}{ccc}0 & 0 & c \\ 0 & 0 & 0 \\ c & 0 & 0\end{array}\right), \quad \mathrm{P}_{\mathrm{E} 1(\mathrm{y})}=\left(\begin{array}{ccc}0 & 0 & 0 \\ 0 & 0 & c \\ 0 & c & 0\end{array}\right), \quad \mathrm{P}_{\mathrm{E} 2}=\left(\begin{array}{ccc}d & d & 0 \\ d & -d & 0 \\ 0 & 0 & 0\end{array}\right)$,

A1(z) represents singly degenerate longitudinal optical (LO) modes which attribute to displacement of the zinc and oxygen atoms along c axis of the unit cell, whereas E1 and E2 modes are doubly (twofold) degenerate transverse optical modes and its defines the displacement of the zinc and oxygen atoms perpendicular to c axis [7]. The constant a,b,c and d are material 
dependent parameters. Hence, to investigate and understand, in-situ evolution of zinc to zinc oxide, UVRRS and PL techniques were opted. UVRRS allows to probe the electron-phonon interaction available in the $\mathrm{ZnO}$ nanostructures. Moreover, inter-band transitions and their effects on the light emission can be directly probed and confirmed by PL spectroscopy. Various reports have been published on the UVRRS of $\mathrm{ZnO}$ nanostructures synthesized by chemical and physical methods. However, no prior report is available on the UVRRS analysis of the $\mathrm{ZnO}$ nanoparticles synthesized using the arc-exploding technique. Understanding vibrational modes and its interactions with free carriers available in $\mathrm{ZnO}$ synthesized by AET, would give a broad impact on the quality of AET synthesized nanoparticles. It is worth to mention that the technique opted for synthesis does not involve any toxic chemical and stabilizer to obtain water stable $\mathrm{ZnO}$ nanoparticles suspension. The research demonstrates the evolution of $\mathrm{ZnO}$ nanoparticles from zinc nanoparticles as a function of ultra-sonication at $30^{\circ} \mathrm{C}$.

\section{Experimental}

Zinc oxide nanoparticles were synthesized using electric arc-exploding method in distilled water medium. In the present method, high purity zinc granules with a diameter of $\sim 3 \mathrm{~mm}$ were used as anode and zinc wire (purity 99.5\%) of $0.5 \mathrm{~mm}$ diameter, procured from Alfa Aesar materials, served as cathode [5]. Two 12 volts VRLA (valve-regulated lead-acid) batteries (Werker, WKA129F2) in series were used to obtain 24 volts potential difference between the zinc electrodes with the maximum current limit of 2.7 A. The arcing was carried out manually at room temperature by shorting the two zinc electrodes in distilled water medium kept in a $200 \mathrm{ml}$ beaker. The process of zinc arc-exploding results in the formation of a black colored nanoparticles suspension which was subsequently allowed to undergo an accelerated oxidation process to $\mathrm{ZnO}$ nanoparticles using ultra-sonication. Finally, the transformed suspension was 
subjected to 70 degree temperature in order to obtain powders of $\mathrm{ZnO}$ nanoparticles. The phase purity of the powders were examined by X-ray diffraction (using Regaku, D/max, Ultima II) studies. A complete phase evolution of $\mathrm{Zn}$ to $\mathrm{ZnO}$ nanoparticles was probed in situ as a function of time employing UVRSS and PL techniques using Jobin-Yvon T64000 microspectrometer. UV absorption measurement was performed using a Perkin Elmer UV-Vis Spectrometer.

\section{Results and discussion}

In order to understand the evolution of wurtzite structure from zinc to zinc oxide in detail, the lattice dynamics studies were performed as a function of time. The evolution time is referred to as sonication time for which the suspension of $\mathrm{Zn} / \mathrm{ZnO}$ nanoparticles was subjected to UVRRS and PL spectra recorded in situ as a function of sonication time are shown in Fig. 1-I (a-q) and Fig 1-II I (a-q), respectively. It was observed from Fig. 1-I(a) that as exploded ( $\mathrm{t}=0 \mathrm{~min}$ ) particles (Zn metal) do not give any UVRRS corresponding to $\mathrm{ZnO}$ nanoparticles. However, the onset of $\mathrm{ZnO}$ phase was observed by the evolution of the peak at $574 \mathrm{~cm}^{-1}$ after one minute of ultrasonication. It is to be mentioned that the intensity of this peak is very low, which may be attributed to the surface oxidation of $\mathrm{Zn}$ nanoparticles resulting in $\mathrm{ZnO}$ at $\mathrm{Zn}$ core shell structure. On the other hand, no significant feature of PL band edge emission around $380 \mathrm{~nm}$ is seen up to 5 min. sonication time (Fig. 1-II(a-e). A broad hump was observed for up to 5 min of sonication time that may be attributed to emission due to disordered surface oxides. Furthermore, on increasing the sonication time, the evolution of second Raman peak at $1148 \mathrm{~cm}^{-1}$ was oserved at 3 min (Fig. 1-I(d). No change was observed in the Raman spectra for 4 min (Fig. 2(e) and $5 \mathrm{~min}$

(Fig. 2f) sonication time and the origination of a third distinct peak at $1722 \mathrm{~cm}^{-1}$ became prominent at 7 min sonication time as shown in Fig. 2(g). Further increasing the sonication time, enhancement in the intensities of all peaks was observed which is indicative of further oxidation 
of $\mathrm{Zn}$ to yield $\mathrm{ZnO}$. The PL spectra also indicate the evolution of the peak at $380 \mathrm{~nm}$ with sonication time (Fig. 1-II (f-q)) attributed to the band edge transition of $\mathrm{ZnO}$ nanostructures.
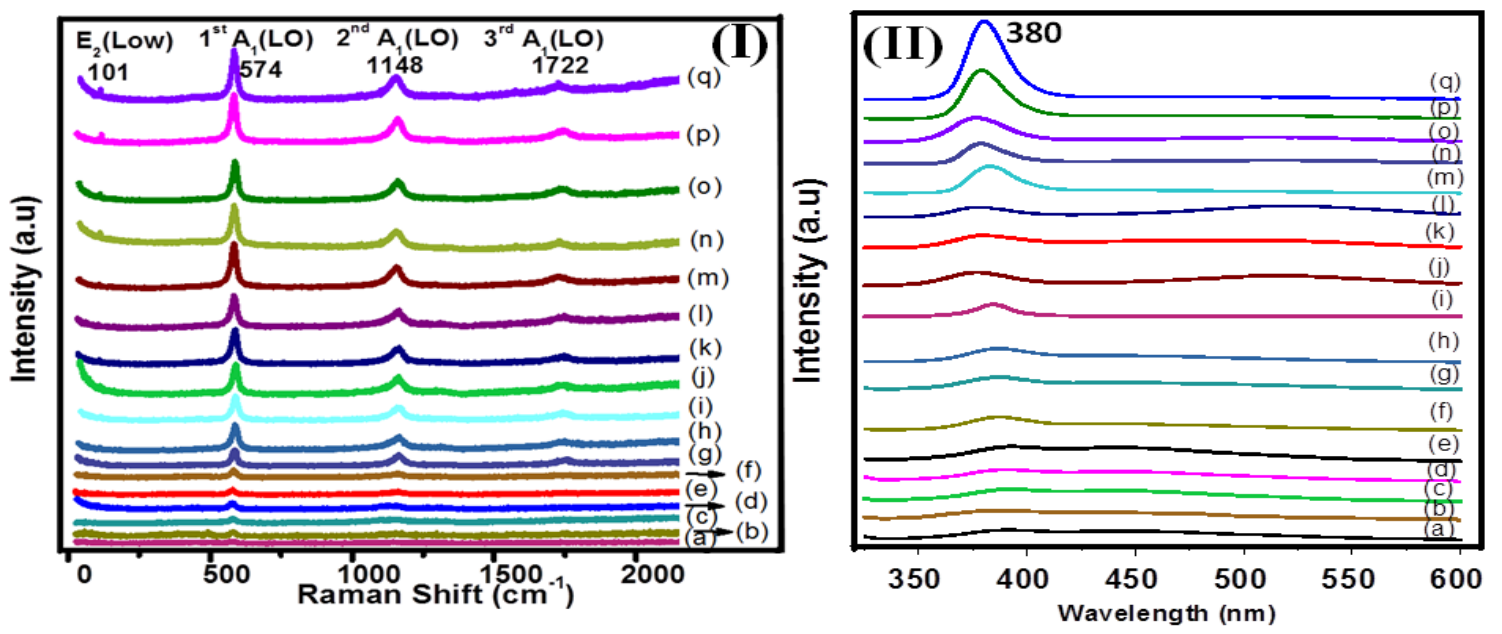

Fig.1. (I) UV Resonance Raman spectra and (II) Photoluminescence spectra of $\mathrm{ZnO}$ nanoparticles as a function of ultra-sonication time (a) $0 \min ($ b) $1 \min$ (c) $2 \min$ (d) $3 \min$ (e) 4 $\min (\mathrm{f}) 5 \min (\mathrm{g}) 6 \min (\mathrm{h}) 7 \min (\mathrm{i}) 8 \min (\mathrm{j}) 9 \min (\mathrm{k}) 10 \min (\mathrm{l}) 15 \min (\mathrm{c}) 20 \min (\mathrm{m}) 40$ $\min (n) 60 \min (o) 120 \min (p) 300 \min (q) 600 \min$.

$\mathrm{X}$-ray diffraction (XRD) pattern recorded for the as synthesized $\mathrm{ZnO}$ nanoparticles showed Bragg reflections corresponding to wurtzite structure (space group $P 6_{3} m c$ ) (Fig. 2(a)). All reflections were Miller indexed using JCPDS card no 36-1451. The lattice parameters of the oxide material were estimated to be $a=b=3.30 \AA$ and $c=5.21 \AA(\mathrm{c} / \mathrm{a}=1.57)$ [8]. The average crystallite size was estimated to be $\sim 14 \mathrm{~nm}$ using Scherrer`s equation. The wurtzite crystal structure generated using the estimated lattice parameters from XRD pattern and Wyckoff position $2 b(x=1 / 3, y=2 / 3, z)$ (Fig. 2(a)). The values of ' $z$ ' for zinc and oxygen atoms were taken as 0 and 3/8, respectively [9]. The UV-absorption spectra recorded on the $\mathrm{ZnO}$ suspension showed the band edge transition at $375 \mathrm{~nm}$. The band gap of the nanoparticles was estimated to be $3.3 \mathrm{eV}$. This is in fairly good agreement with the results for PL analysis (Fig. 1-II(q)). 

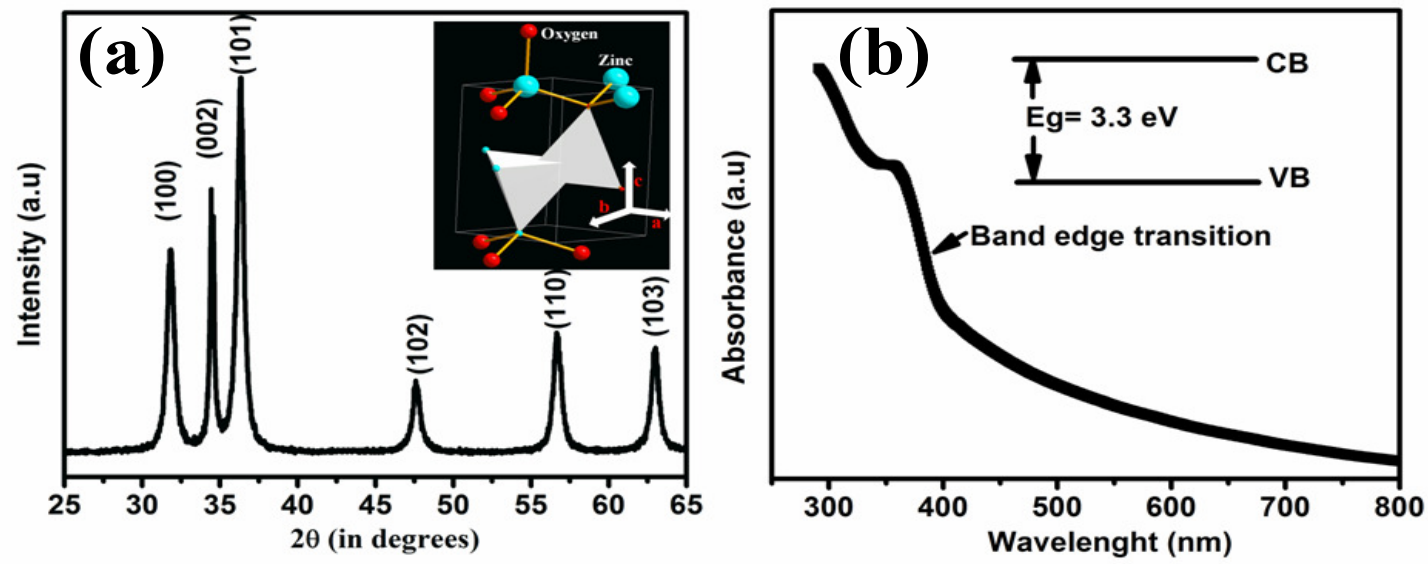

Fig. 2. (a) XRD pattern of $\mathrm{ZnO}$ nnaoparticles with inset showing wurtzite crystal structure (b) UV absorption of $\mathrm{ZnO}$ nanoparticles.

The observed Raman peaks depicted in Fig. 3(a) correspond to multiple harmonics of $\mathrm{A}_{1}$ longitudinal phonon (LO) modes which originated due to RRS in $\mathrm{ZnO}$ nanoparticles. The observed $\mathrm{A}_{1}(\mathrm{LO})$ modes were fitted by keeping Lorentzian centres at $\mathrm{n} \hbar \omega_{\mathrm{LO}}(\mathrm{n}=1,2$ and 3$)$ and are shown in Fig. 3(a). The values of multiple LO modes are given as $574 \mathrm{~cm}^{-1}\left(1 \omega_{\mathrm{LO}}\right), 1148 \mathrm{~cm}$ $1\left(2 \omega_{\mathrm{LO}}\right)$, and $1722 \mathrm{~cm}^{-1}\left(3 \omega_{\mathrm{LO}}\right)$ corresponding to the $1^{\text {st }}, 2^{\text {nd }}$ and $3^{\text {rd }}$ harmonics of $\mathrm{A}_{1}(\mathrm{LO})$ modes, respectively. The origin of these nLO lines is attributed to the displacement of zinc and oxygen atoms parallel to the c-axis of the wurtzite structure as shown in Fig. 3(b). The observed enhanced UVRRS is attributed to electron - phonon scattering given by Frohlich interaction between the phonon modes and the hot excitons [10]. The evolution of multiple LO modes with its variation in the intensity can be understood using the 'cascade' model [11], wherein, the creation of excitons is effected by means of an incident photon absorption (h$\left.\omega_{\mathrm{I}}\right)$ followed by the non-radiative relaxation of excitons in to lower energy levels resulting in the successive emission of LO phonons. The annihilation of these excitons with emission of phonons is given by $\left(\hbar \omega_{\mathrm{sn}}=\hbar \omega_{\mathrm{I}}-\mathrm{n} \hbar \omega_{\mathrm{LO}}\right)$. The intensity ratio of the observed peaks were estimated to be $\mathrm{I}_{2 \mathrm{LO}} / \mathrm{I}_{1 \mathrm{LO}}$ 
$(0.46)>\mathrm{I}_{3 \mathrm{LO}} / \mathrm{I}_{2 \mathrm{LO}}(0.35)$ indicating dissociation of hot excitons at higher order $\mathrm{LO}$ harmonics [12].

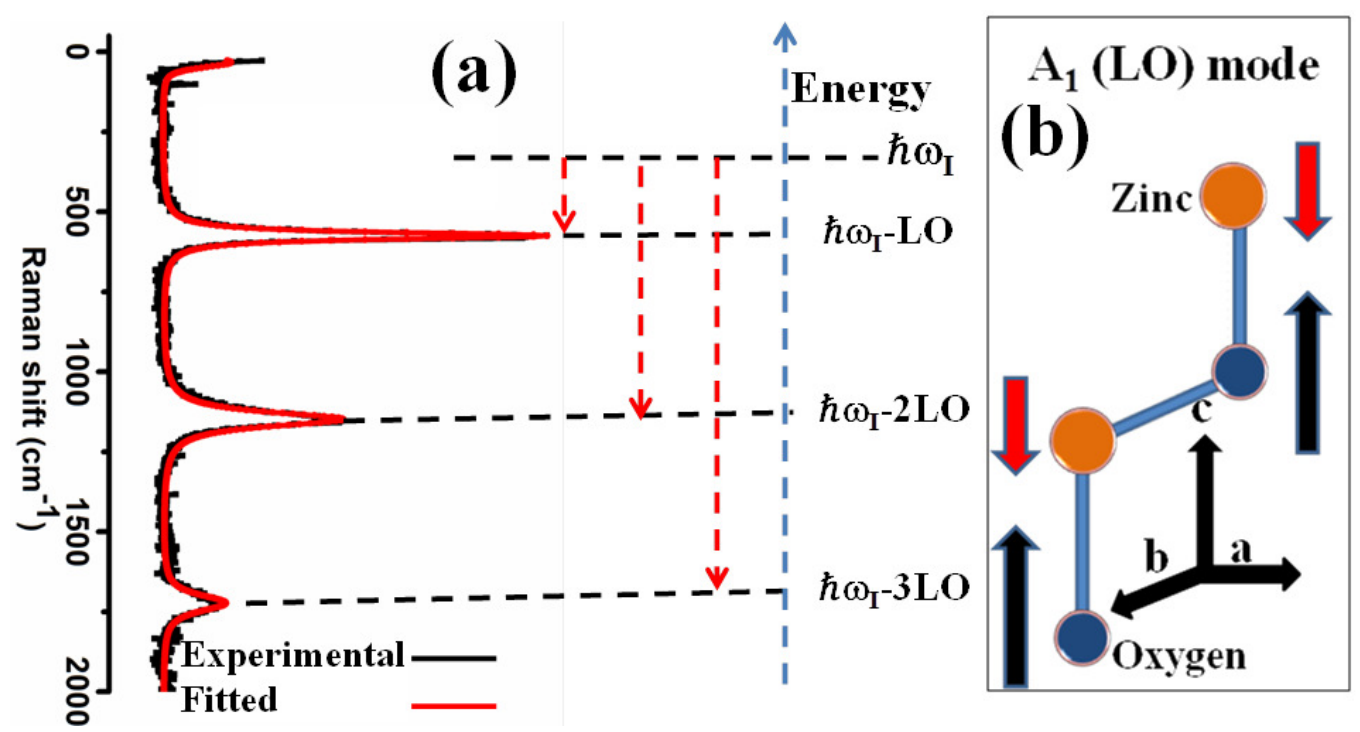

Fig. 3 (a) Schematic of process of multiple- $\mathrm{A}_{1} \mathrm{LO}$ phonons in the RSS (b) corresponding phonon dynamics in the wurtzite structure parallel to c-axis.

\section{Conclusions}

We have successfully demonstrated synthesis of $\mathrm{ZnO}$ nanostructures using AET. The in situ evolution of $\mathrm{Zn}$ to $\mathrm{ZnO}$ nanostructures is successfully demonstrated using the time dependent Raman and photoluminescence studies. XRD and spectrophotometric studies further confirm the formation of wurtzite structure and provide a band gap of $\sim 3.3 \mathrm{eV}$, respectively. Homogeneity of the synthesized nanostructures is demonstrated using the UVRRS and PL. The strong multiple $\mathrm{A}_{1} \mathrm{LO}$ phonons observed owing Frohlich interaction between the LO phones and hot excitons is explained using the cascade model. The suppression in nLO intensity in UVRRS is attributed to the dissociation of the hot excitons at higher order harmonics. 


\section{Acknowledgement}

Authors would like to acknowledge the financial support from DoD \#W911NF-11-1-0204 to

carry out this research work. S. P. P. and R.K.K. would like to acknowledge the financial assistance under Grant No: NSF-EFRI RESTOR \# 1038272.

References:

1. Ozgur U, Alivov YI, Liu C, Teke A, Reshchikov MA, Dogan S, Avrutin V, Cho SJ, Morkoc H, J. Appl. Phys. 2005; 98:041301-1-103.

2. Djurisic AB, Ng AMC, Chen XY, Progress in Quantum Electronics 2010; 34:191-259.

3. Radzimska AK, Jesionowski T, Materials 2014; 7: 2833-81.

4. Singh NK, Alqudami A, Annapoorni S, Phys. Status Solidi A 2010; 207:2153-58.

5. Singh NK, Medwal R, Annapoorni S, Mater Sci 2014; 49:8386-93.

6. Arguello CA, Rousseau DL, Porto SPS, Phys. Rev. 1969; 181:1351-63.

7. Calleja JM, Cardona M, Phys. Rev. B, 1977;16:3753-61.

8. Maurya A, Chauhan P, Mishra SK, Srivastava RK, J. Alloys and Compd. 2011; 509: 843340.

9. Escudero R, Escamilla R, Solid State Communications 2011; 151: 97-101.

10. Ursaki VV, Tiginyanu IM, Zalamai VV, Rusu EV, Emelchenko GA, Masalov VM, Samarov EN, Phys. Rev. B 2004; 70:155204-1-8.

11. Martin RM, Varma CM, Phys.Rev.Lett. 1971; 26:1241-44.

12. Cerqueira MF, Vasilevskiy MI, Oliveira F, Rolo AG, Viseu T, Ayres deCampos J, Alves E, Correia R, J. Phys.: Condens. Matter 2011; 23:334205-1-6 
Figure Picture
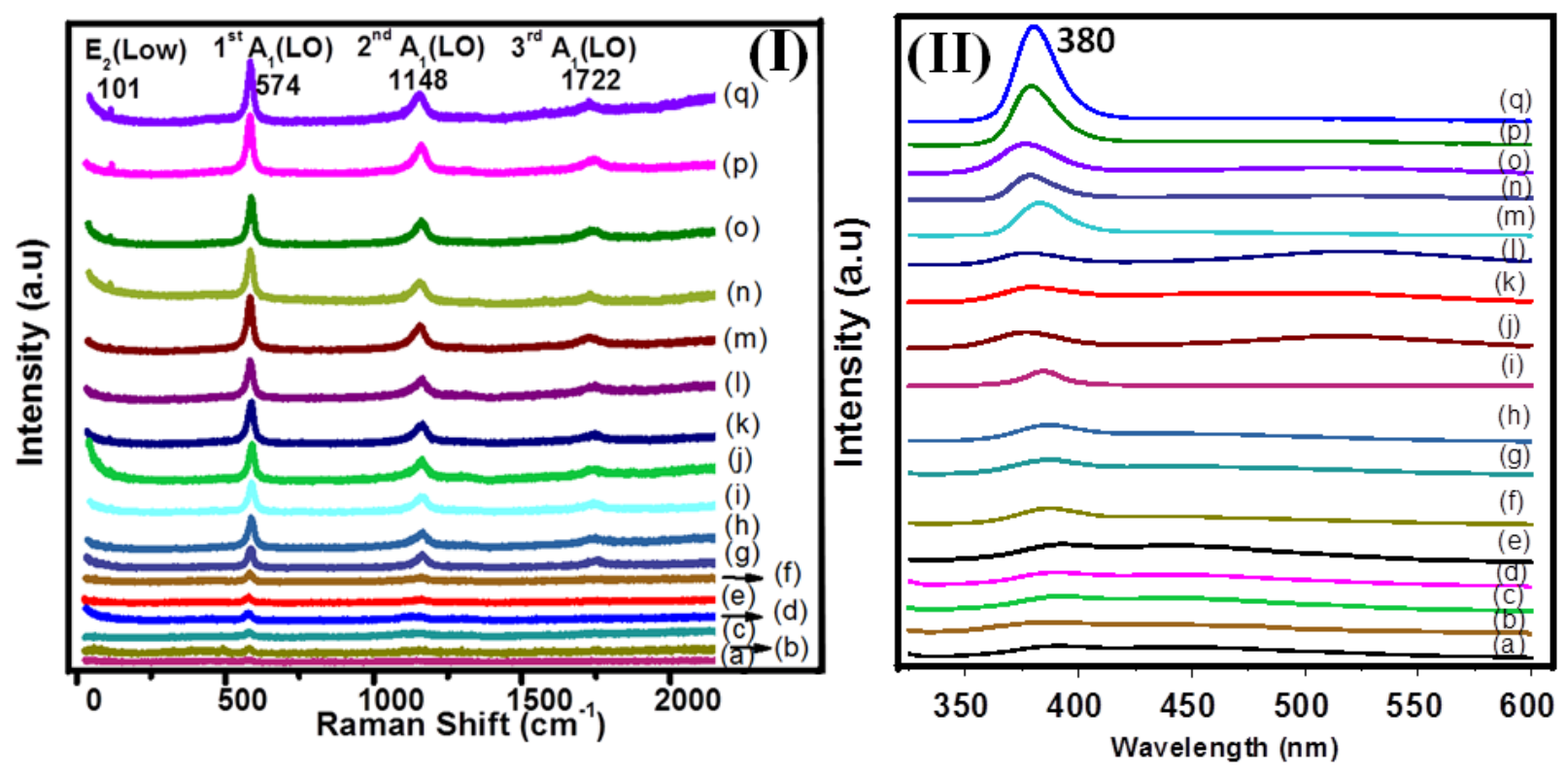

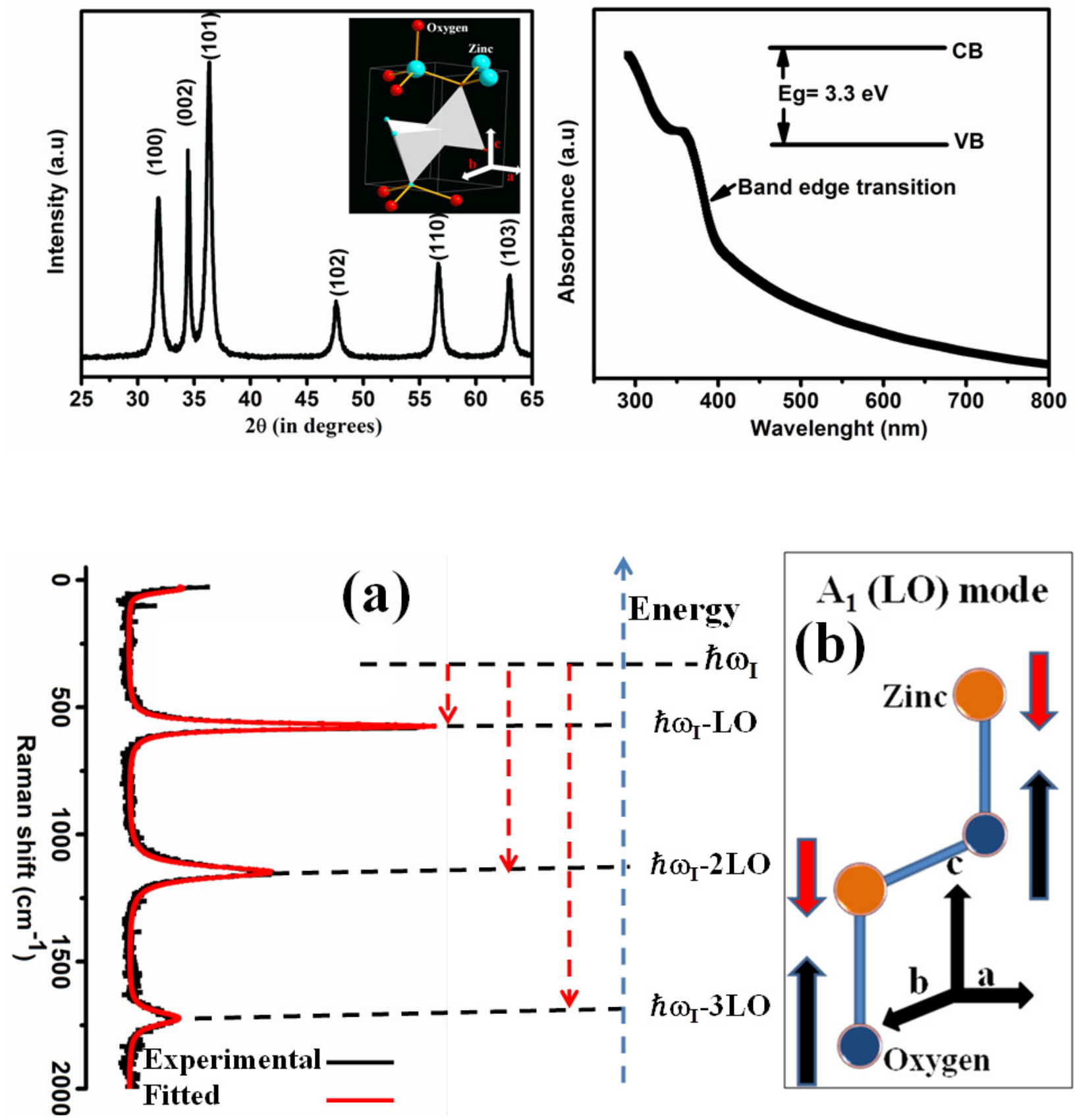


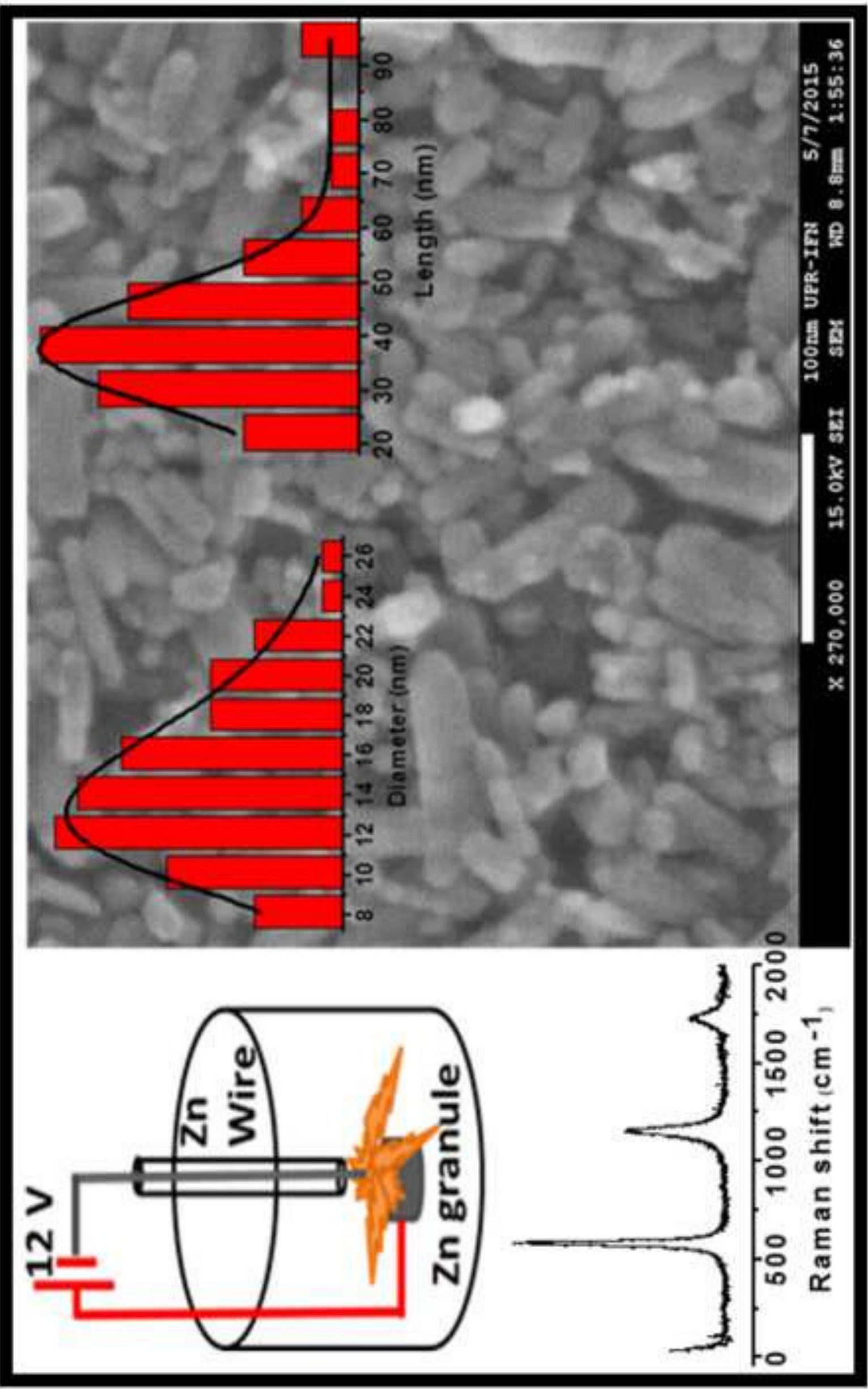

